\title{
QUÉ NUEVA INTERVENCIÓN \\ PARA QUÉ NUEVAS REALIDADES FAMILIARES: \\ EL PROBLEMA DE LA DIVERSIDAD FAMILIAR EN LA SOCIEDAD ESPAÑOLA Y SUS IMPLICACIONES PARA EL TRABAJO SOCIAL
}

\author{
IVÁN RODRÍGUEZ PASCUAL
}

Departamento de Sociología y Trabajo Social. Universidad de Huelva.

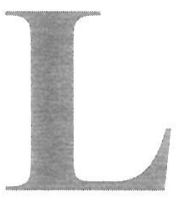

\section{RESUMEN}

a diversidad familiar se considera un indicador de modernización social, pero lleva aparejada serias implicaciones en lo concerniente al desarrollo de políticas sociales destinadas a la protección a la familia. Teniendo en cuenta que en España no existe nada que pueda llamarse con propiedad "política de protección a la familia", el fenómeno de la diversidad familiar añade un punto de complejidad al escenario de la política social y el desempeño de la labor asistencial. Reivindicamos una mayor atención para las familias y proponemos que el desarrollo de esa política de protección a la familia, ausente hoy día, comience por el respeto de las diferentes formas familiares y sus circunstancias.

Abstract: Family plurality is considered an indicator of social change towards modernization, but it implies main aspects concerning the development of social policies for the family. Starting in the fact that there is no such family policy in Spain, this phenomenon adds complexity to social and political scene. We demand more attention paid to families as well as a development of this family policy that should rest in the respect for different family types and their circumstances.

Palabras clave: familia-politica social-diversidad familiar / family-social policy-family plurality. 


\section{INTRODUCCIÓN: A VUELTAS CON LA DIVERSIDAD}

Viene siendo usual utilizar la cuestión de la diversidad familiar como un indicio, más o menos fiable, de la presencia de una tendencia hacia la modernización de las formas familiares o, lo que es lo mismo, de la presencia de eso que llamamos (quizás muy a la ligera) "progreso".

En este texto vamos a defender una posición sensiblemente diferente. En nuestra opinión, la diversidad familiar constituye una manifestación de un cambio social más general que afecta a otras instituciones sociales, tanto como a los fundamentos organizativos de la sociedad misma, y que difícilmente puede ser calificada a priori como buena o perjudicial para el conjunto de las relaciones sociales desde el mismo momento en que participa de ambas naturalezas. En cualquier caso, no compartimos la imagen, presente de manera latente en muchos textos de sociología de la familia, de las nuevas formas familiares como enfrentadas u opuestas a la familia nuclear biparental tradicional, sino emanando en muchas ocasiones de ella, o mimetizando sus formas y costumbres. De la misma manera, pretender encontrar en el modelo nuclear de convivencia un patrón inmutable, bendecido por la durabilidad de lo tradicional, sería abundar en un error mil veces cometido. Como nos enseña Anthony Giddens (2001) la mayor parte de las cosas a las que aplicamos el calificativo de "tradicional" son relativamente modernas y novedosas, resultado de una construcción social.

Por tanto, entenderemos que la diversidad familiar es, simplemente, una tendencia de cambio que amplía la legitimidad a un rango más o menos amplio de estructuras familiares, creando nuevas necesidades y demandas, pero que no pone en peligro el orden social ni resulta de una temida (tanto como poco probable) desinstitucionalización familiar o crisis de la familia. Al mismo tiempo, entendemos que el panorama futuro vendrá marcado por la existencia de considerables cuotas de diversidad junto a formas decididamente "tradicionales", amén de otros estilos de vida que quizás hoy todavía ni siquiera conozcamos.

A continuación intentamos explicitar en qué consiste tal diversidad familiar en el contexto de la sociedad española, para seguidamente abordar la cuestión de las nuevas necesidades surgidas de tal diversidad y su relación con la labor del trabajador social orientada a la intervención. De esta manera, se intenta al mismo tiempo desligar la práctica del trabajo social de su conceptualización como profesión dirigida exclusivamente a colectivos en riesgo social. Precisamente, tal imagen emanaba de una sociedad típicamente familista en la que la familia (y dentro de ella las mujeres) ocupaba un espacio asistencial importante como proveedora de servicios fundamentales de atención a distintos grupos de población 
(niños, ancianos, enfermos, discapacitados, etc.), y la atención estatal quedaba reservada para otros casos de exclusión. Entendemos que la lógica del cambio social y familiar dificulta cada vez más esta tarea si depende excluidamente de las redes familiares, por lo que la aparición de estas nuevas formas familiares representa un punto de inflexión a la hora de plantear tanto la naturaleza de la práctica profesional de los trabajadores sociales como el papel de los servicios sociales y el Estado de Bienestar ante las demandas y necesidades familiares.

\section{II. ¿DE QUÉ HABLAMOS CUANDO HABLAMOS DE DIVERSI- DAD FAMILIAR? EL PROBLEMA DE LA MODERNIZACIÓN DE LAS FORMAS FAMILIARES EN EL CONTEXTO DE LA SOCIEDAD ESPAÑOLA}

Efectivamente, lo que convierte al asunto de la diversidad familiar en una cuestión candente es su valor como "indicador" de progreso y modernización. Ésta tendría un punto álgido como resultado de una supuesta desinstitucionalización familiar provocada por la liberalización respecto de las costumbres y los modelos familiares tradicionales, así como de una interpretación de las relaciones familiares claramente individualista, donde la familia es, antes que nada, un problema de libre elección personal relacionado con la afectividad y los avatares biográficos de los individuos, como queda bien ejemplificado entre los defensores de la llamada "segunda transición demográfica" (Van De Kaa, 1987).

Por supuesto, el estudio de la diversidad o pluralidad de las formas familiares no puede desligarse de la contemplación de los cambios acelerados que ha sufrido esta institución en el contexto de la sociedad española. Así, cada vez en mayor medida, los indicadores muestran que tratamos con familias menos autoritarias, con más espacio para la individualidad y la reivindicación de los intereses personales. También más íntimas y privadas, si entendemos esto último como que proveen de un refugio frente al espacio público, así como más tolerantes respecto de la cuestión de la convivencia entre generaciones. Ya es frecuente la referencia al matrimonio-contrato, desafortunado término que alude a la relativización de la importancia de la unión matrimonial en función de la consecución de la felicidad personal y conyugal (Alberdi, 1993b; Del Campo 1991; CECS, 1995; Pastor Ramos, 1997). O la consideración de la familia como ámbito de la libertad personal, donde se desarrollan estilos de socialización más cercanos al apoyo y la comunicación intrafamiliar que al esquema puramente autoritario y lanzadera para la construcción de biografías individuales relativamente alejadas de ella 
(Alberdi, 1995; De Miguel, 1994; Flaquer y Soler, 1990). La ruptura, en fin, con la imagen de la familia como obligación vital, de los vínculos matrimoniales indisolubles y duraderos, vinculados a obligaciones tales como la obtención de descendencia o la sumisión de la mujer al marido (Cruz, 1995; Iglesias de Ussel, 1994; 1998; Pastor Ramos, 1997).

Sin embargo, si hay algo cierto en el panorama de la vida familiar de las sociedades industrializadas es que el concepto "diversidad" resulta vacío y demasiado abstracto si no se le dota de contenido. Dicho de otra forma, distintas sociedades han experimentado cambios en las formas familiares que no necesariamente han de ser equivalentes o convergentes.

Para el caso contamos con la ya conocida caracterización de los modelos familiares en Europa (Requena, 2001) donde la complejidad del cambio de las formas familiares se hace evidente. Mientras que las sociedades escandinavas y del norte de Europa parecen haber experimentado una ruptura radical respecto al modelo familiar tradicional, con una natalidad extramatrimonial acusada y una fuerte tendencia a construir vínculos matrimoniales efímeros y formas de cohabitación alternativas al matrimonio, las sociedades mediterráneas y centroeuropeas siguen posicionándose más cerca de un modelo mixto en el que la familia nuclear sigue gozando de buena salud, por más que se transformen los estilos de vida y los papeles que acoge en su seno. En este sentido, caracteriza a la sociedad española una mayor complejidad de los hogares (también más numerosos) y una incidencia relativamente baja de eso que se ha dado en llamar "nuevas formas familiares".

En realidad, la misma noción de "nuevas" formas familiares podría resultar engañosa en la medida en que la mayor parte de ellas son antiguas y bien conocidas ${ }^{1}$. Quizás lo que sí sea novedoso y haya inducido a error es el escenario en el que estas nuevas formas familiares se insertan: de una sociedad marcada por el predominio justificado en términos ideológicos de un único tipo de familia, que habitualmente silenciaba o disfrazaba de patológico la existencia de otras formas de organización familiar, a otra en la que la tolerancia social hacia ellas es amplia y cada vez más extendida aspirando a gozar, incluso, de un tratamiento legal que no sea discriminatorio. No obstante, muchos de los nuevos fenómenos que en este ámbito se están sucediendo son difíciles de detectar y sobre todo de cuantificar; las quejas sobre la mala calidad de las estadísticas oficiales sobre los hogares y las familias han sido frecuentes en el marco de la sociología española -véase si no, por ejemplo, el conocido

1 Véase, a modo de ejemplo, la descripción de la "barraganeria" que ofrece el profesor Felipe Morente (1996). 
manual de Iglesias de Ussel (1998) - y a la hora de intentar cuantificar la incidencia de estas nuevas familias lo es aún más. A la cuestión de por qué hablamos de "nuevas formas familiares" para referirnos a estructuras y modos de vida preexistentes podemos responder con los argumentos del profesor Díaz Becerril (2004):

- En primer lugar, por el incremento de su presencia cuantitativa y la rapidez con que crece su número en los últimos años sobre el conjunto de las formas familiares, por más que la familia tradicional (nuclear, biparental, heterosexual) goce de buena salud en este sentido. Esto debe completarse con el hecho de su difusión a lo largo de diferentes sectores sociales (con lo que en muchos casos pierden su particularidad como comportamiento de un grupo determinado) y la tolerancia social con que son contempladas por el conjunto de la sociedad ${ }^{2}$.

- No es menos importante el efecto que estas nuevas formas familiares han tenido en la fragmentación y diversificación del llamado ciclo de vida familiar, que asume un cierto componente de incertidumbre toda vez que los individuos entran y salen de diferentes formas de convivencia en función de los avatares de su biografía personal.

- Precisamente, esto guarda relación con el hecho de que, hoy día, resulta una nota característica de las nuevas formas familiares el considerarlas viables y capaces de mantenerse por sí mismas, y no simplemente una forma residual o provisional de convivencia.

- Como consecuencia de su mayor aceptación, constituye también un elemento novedoso el que la diversidad familiar exista de manera pública, y no únicamente como manifestación privada y oculta. $\mathrm{Su}$ publicidad es, al mismo tiempo, condición necesaria que explica el fuerte impulso registrado en la sociedad española hacia el reconocimiento legal de su situación, al mismo nivel que las familias tradicionales, buscando claramente un objetivo de legitimación.

- Por último, es significativo que su origen ya no provenga exclusivamente de circunstancias externas o imprevistas en la vida de los individuos (mortalidad sobrevenida, por ejemplo), sino que sean fruto de la voluntad personal de los mismos y de un intento de construcción de biografías personales.

2 A este respecto, los estudios que viene realizando periódicamente el Centro de Investigaciones Sociológicas nos proveen de suficientes datos significativos. Baste citar, por ejemplo, la aceptación con que la población española acoge reconocimiento legal de las parejas homosexuales (un $57 \%$ se muestra de acuerdo con su existencia, del que el $85 \%$ aceptaría un matrimonio civil homosexual) o las parejas de hecho en general (76\%) (CIS, 1997), buena muestra del cambio actitudinal operado en nuestra sociedad en un breve lapso de tiempo que va desde el final del régimen franquista hasta hoy dia. 
Precisamente, un intento de cuantificación de esta nueva realidad familiar es lo que presentamos en la figura siguiente. Hemos utilizado preferentemente fuentes censales o de probada fiabilidad, indicando el año al que se refiere la información, aunque la explotación "por goteo" que el INE está realizando del censo de población de 2001 nos ha privado de completarlo actualizado en su totalidad. Como se ve, se han distinguido dos grandes grupos de nuevas formas familiares, unipersonales y pluripersonales, y dentro de éstas hacemos referencia a los hogares unipersonales, las familias monoparentales, las reconstituidas y las parejas de hecho. A éstas habría que unir, para completar el panorama de la diversidad, las familias nucleares tradicionales, así como las familias nucleares que han comenzado a cambiar hacia un modelo de doble carrera tras la incorporación plena de ambos miembros de la pareja al mercado laboral.

Figura 1: Las nuevas formas familiares (\% sobre total de hogares) (se indica, junto a la estimación, la fuente de procedencia)

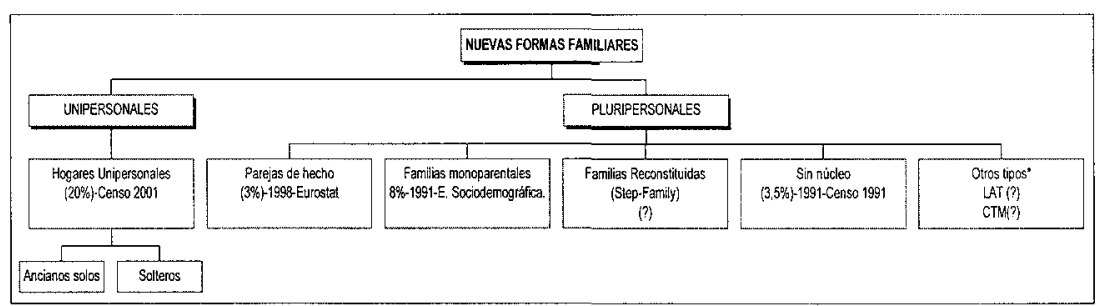

* LAT: Living Apart Together (parejas sin convivencia residencial)

$\mathrm{CM}$ : commuter Marriage (matrimonios de fin de semana)

¿Qué es lo que puede decirse de estas formas novedosas de "estar" y vivir en familia? Dependiendo de la modalidad de la que estamos hablando, poco o algo más, pero siempre menos de lo que nos gustaría saber.

Algunas de estas formas familiares han atraído buena parte de la atención investigadora y de la opinión pública. Sin duda es el caso de las llamadas familias monoparentales. Son ya numerosos los estudios dedicados al tema desde una perspectiva sociológica de entre los cuales citamos sólo los más recientes (Barbadillo, 1995; Donald, 1994; Fernández y Tobío, 1988; González, 2000; Iglesias de Ussel, 1998; Moreno, 2000; Sarrible, 1996; Tobío y Fernández, 1999). Sabemos que, si bien no son nuevas, ha cambiado sensiblemente su composición al alterarse la fuente de este tipo de núcleos familiares acusando el crecimiento de la ruptura matrimonial de los últimos años; también presentan distintos perfiles demográficos y socioeconómicos, sin olvidar la ya mencionada tolerancia (con reservas) por parte de la sociedad en general hacia este tipo de familias. Por otra parte, como suele ocurrir 
con los sucesos novedosos, nos encontramos con ciertos problemas a la hora de definir sus contornos y cuantificarlos. La fórmula escogida por los investigadores apunta a considerar como monoparental sólo a los adultos con hijos menores de 18 años a cargo. En cualquier caso, el número que se maneja se encuentra en torno al 8 ó $10 \%$ del total de hogares, dependiendo de la estimación, lo que probablemente involucra a más de dos millones de personas. En lo fundamental, son hogares abrumadoramente encabezados por mujeres, procedentes cada vez más de rupturas matrimoniales y fuertemente afectados por procesos de empobrecimiento y merma de recursos. A ello hay que añadir que la sociedad no ha desarrollado todavía una institucionalización eficiente respecto a las necesidades de estas familias y sigue funcionando sobre la plataforma de la biparentalidad (Iglesias de Ussel, 1998). Durante mucho tiempo este tipo de familias fue considerado desde la perspectiva normativa como una forma anormal, desviada o patológica. Quizás por ello, los núcleos monoparentales encuentran una dificultad especial para desenvolverse en una sociedad que, si bien afirma tolerarlos y comprenderlos, ofrece pocas facilidades a la hora de intentar suavizar o paliar los problemas propios de su situación, a saber: recursos escasos; conflictividad conyugal; exposición a un proceso de separación que en muchas ocasiones resulta traumático o estresante; integración laboral de las mujeres con hijos a cargo; necesidad de protección social específica, etc. Esta aparente situación de semiexclusión a la que se enfrentan muchos adultos con menores a cargo se agravará si tenemos en cuenta que el ritmo de la ruptura matrimonial en España es creciente y sostenido, lo que permite suponer que el peso relativo de este tipo de núcleos familiares no puede menos que seguir aumentando, y con ello los problemas específicos asociados al fenómeno.

Por contra, es poco, nada o casi nada lo que sabemos sobre otro tipo de núcleo familiar como son las conocidas en el ámbito anglosajón con el nombre de step-family, y aquí como familias reconstituidas. Ignoradas por la estadística y poco explorada su situación en la sociedad española por los científicos sociales, tan sólo podemos suponer que crecerán a un ritmo parecido al de las propias familias monoparentales, dado que resultan cada vez más frecuentes (aunque más en los varones) las segundas nupcias tras los procesos de ruptura (Iglesias de Ussel, 1994; 1998).

Simultáneamente, tenemos un crecimiento intenso de hijos tenidos fuera del matrimonio que no puede interpretarse únicamente como consecuencia de embarazos no deseados, sino también como parte de una nueva concepción de la maternidad fuera de estructuras biparentales. Éstos pasaron de representar apenas un 3\% a finales de los años 70 a 
más de 16 de cada 100 nacidos en 1999 , de acuerdo con las cifras proporcionadas por el propio Instituto Nacional de Estadística (2002).

En este sentido, no debe pasarse por alto el crecimiento de la cohabitación como opción de vida en pareja en estos últimos años. Todavía sigue siendo, como ya se ha apuntado, muy minoritaria, aunque gana terreno en los ambientes urbanos y entre los jóvenes. La Encuesta Sociodemográfica, producida por el INE y referida a 1991, cifraba su existencia en apenas un $1,1 \%$ del total de hogares (menos de un $1 \%$ de la población), un dato que probablemente haya quedado desfasado por más que siga siendo significativo. Lo que los datos indican es que la cohabitación en el contexto de la sociedad española no explica la reducción registrada de la nupcialidad y parece situarse más como una situación previa al matrimonio para los más jóvenes antes que una alternativa consolidada al mismo (Castro, 1999). Precisamente, también es poco lo que podemos decir respecto a este tipo de uniones protagonizadas por miembros del mismo sexo. Que la sociedad tiende a tolerarlas cada vez más es claro, pero ¿hasta qué punto?

Nada hemos hablado de los hogares unipersonales, que constituyen ya una cuarta parte del total y son la forma de vida de unos tres millones de personas. En parte porque queda claro que son uno de los tipos que más ha crecido recientemente, aunque buena parte de este crecimiento se ha debido a un fuerte proceso de envejecimiento que condena a muchos ancianos a una vida solitaria en condiciones de exclusión. Los datos del censo de 2001 revelan que la inmensa mayoría de los hogares unipersonales (concretamente más del 60\%) están ocupados por personas mayores de 65 años. Sin embargo, también son muchos (el INE los cifra en unos 190.000 por debajo de los 34 años) los hogares compuestos por personas jóvenes que se benefician del cambio en la percepción social de la soltería, cuando las palabras "solterón" y "solterona" han caído en desuso y ser soltero puede no ser un fracaso en el ciclo vital.

En cualquier caso, todas estas formas familiares que habitualmente englobamos en la categoría de "nuevas" dependen para su subsistencia de su capacidad para desenvolverse en un marco legal pero también económico y social que no ha previsto su eclosión y que sigue moviéndose, en el terreno subjetivo del discurso y en el objetivo de los recursos y prestaciones sociales, sobre la base de la biparentalidad y la familiar nuclear en un sentido clásico o tradicional, provocando un serio desajuste entre dicho marco y la aceptación tácita de la pluralidad familiar. Por ello, no es descartable que muchos de los debates políticos de los próximos años en torno al tema de la familia estén protagonizados por la cuestión general de la modernización del sistema legal hacia un mayor reconocimiento (o no) de esta realidad diversa y compleja 
(Pastor, 1997: 382 y ss.), lo cual nos conduce al problema de la articulación de mecanismos suficientes de intervención social que respalden el bienestar de las familias (o de todas las unidades de convivencia con un estatus reconocido en el marco de la sociedad española), que es, en última instancia, el bienestar de la sociedad.

\section{UN MODELO PLURALISTA DE INTERVENCIÓN FAMILIAR PARA UNA "NUEVA" FAMILIA}

Vaya por delante que el núcleo principal de nuevas demandas orientadas a la intervención social mantendrá una relación estrecha con la reivindicación de un marco más flexible y desarrollado de protección a la familia. Una de las peculiaridades de la sociedad española es que, frente a lo que viene siendo común en el contexto de los Estados de Bienestar, ésta ha desarrollado escasas políticas de protección a la familia, y las que se han puesto en marcha no han sabido incorporar en su formulación la realidad de la diversidad de las formas familiares (Alberdi, 1997; Flaquer, 2000; Iglesias de Ussel, 1994; 1998; Meil, 1995). Por ello, la intervención social sobre la familia del futuro vendrá marcada por esta diversidad, sin identificarse estrictamente con ella. Esta última apreciación nace del hecho de que, si bien el apoyo a las familias debe constituir parte integrante del trabajo social, resulta prioritario orientar ésta hacia aquellas formas de convivencia que disfrutan de menor calidad de vida o de problemas relativos a la disponibilidad de los recursos mínimos necesarios para garantizar que se evita su caída en la exclusión social.

Basándonos en estas consideraciones, hemos vuelto a categorizar las llamadas "nuevas formas familiares", junto a las formas "tradicionales", en función de dos criterios que nos parecen suficientemente significativos: a) según el riesgo de exclusión que se estima que corren y, b) según el nivel de demandas novedosas de intervención que, potencialmente, podrían producir. De esta manera, la intersección de ambos criterios marca la población que, en nuestra opinión, debe constituir el objetivo preferente de la intervención social sobre la familia, por aunar un elevado riesgo de exclusión con la presencia de un fuerte potencial de necesidades de intervención.

En esta situación hemos localizado dos formas familiares: los hogares unipersonales habitados por ancianos y las familias monoparentales. Por supuesto, no hemos descartado a las familias nucleares tradicionales en situación de exclusión, pero éstas han sido ubicadas en un cuadrante distinto, ya que el nivel de nuevas demandas potenciales de interven- 
ción que presentan es, al menos en teoría, bajo, al haber sido contempladas, si quiera mínimamente, por el actual marco de intervención.

Tabla 1. Tipología de la situación social y demandas potenciales de las nuevas formas familiares

RIESGO DE EXCLUSIÓN/MENOR CALIDAD DE VIDA Alto $(+)$

Bajo (-)

\begin{tabular}{|c|c|c|}
\hline $\begin{array}{l}\text {-Familias monoparentales } \\
\text {-Hogares unipersonales (ancianos) }\end{array}$ & -Familias reconstituidas (step family) & Alto $(t)$ \\
\hline & & POTENCIALDE \\
\hline -Familias nucleares tradicionales & $\begin{array}{l}\text {-Parejas de hecho } \\
\text {-Hogares unipersonales (jóvenes) } \\
\text {-Familias nucleares (doble carrera) }\end{array}$ & $\begin{array}{l}\text { DE NUEVAS } \\
\text { DEMANDAS DE } \\
\text { INTERVENCIÓN } \\
\text { Bajo }(-)\end{array}$ \\
\hline
\end{tabular}

En otro lugar, constituyendo un campo de intervención para el trabajo social legítimo pero menos acuciante, al menos en un sentido relativo, tenemos a las parejas reconstituidas o step family. A las parejas de hecho y a las familias de doble carrera, así como a los hogares unipersonales ocupados por jóvenes, las hemos caracterizado como formas familiares que generan un nivel más bajo que las anteriores en cuanto a las potenciales demandas de intervención social y se encuentran más alejadas del problema genérico de la exclusión social, dado que en su perfil cuentan con mejores condiciones de vida y un acceso más fluido a los recursos necesarios para que éstas se mantengan en un nivel satisfactorio.

\section{A MODO DE CONCLUSIÓN}

De lo expuesto hasta ahora se deduce que constituiría un error considerar las "nuevas formas familiares" un grupo homogéneo de formas de convivencia necesitado de similares fórmulas de intervención por parte del trabajador social. Todas ellas representan una manifestación particular de vínculos interindividuales que tienen su origen en un cruce entre la voluntad personal y las oportunidades vitales de los individuos en su contexto social. Precisamente, si reconocemos que la diversidad es, ante todo, eso, una multiplicidad de formas de convivencia, no resulta descabellado suponer que el tipo de atención prestado a estas formas de convivencia debe ser igualmente diverso y multidimensional. Esto choca con el panorama de la política familiar española, claramente insuficiente y orientada todavía desde la perspectiva de la familia nuclear tradicional y sus necesidades. 
Esta tipología, como cualquier otra, presenta el problema de que sólo es válida en la que medida en que no es real. Es decir, constituye una abstracción de los problemas concretos y diversos de las familias españolas que no debe interpretarse en un sentido demasiado rígido, sino como guía (flexible y susceptible de perfeccionamiento) para la acción.

Pretendemos clarificar de qué hablamos cuando hablamos de diversidad familiar, al tiempo que reivindicar el campo de la familia (y no sólo las excluidas) como un campo de intervención para el trabajo social, denunciando a la par la situación de desprotección en la que se encuentran la totalidad de las familias españolas. Por ello, defendemos la idea de que a la común reivindicación del desarrollo de una política coherente y exhaustiva de protección social a la familia es necesario añadir una llamada de atención para el trabajo social que identifique plenamente el problema de la diversidad familiar y sus nuevas demandas de intervención como un campo profesional legítimo para la profesión, añadiendo criterios de trabajo suficientemente operativos para ejercer de guía para la intervención.

La aplicación de los criterios aquí designados ha permitido identificar dos nuevas formas de convivencia que parecen candidatas potenciales a caer en la dinámica de la exclusión, y que necesitan de una intervención urgente: concretamente los hogares unipersonales habitados por ancianos y las familias monoparentales. Al tiempo, se ha alertado sobre la posibilidad de que otras nuevas formas familiares generen, en un futuro cercano, importantes demandas de intervención social, como es el caso de las step family, grandes desconocidas en nuestro contexto, y otras que representan una prioridad más baja aunque constituyan una novedad en el panorama de las distintas formas familiares existentes en la sociedad española.

Creemos que una política familiar más acertada debería buscar una cobertura mejor para todas estas formas familiares, partiendo de las situaciones de mayor necesidad y acercándose a las nuevas necesidades del resto de las formas y estructuras de convivencia que hemos presentado a lo largo de este texto.

\section{BILIOGRAFÍA}

ALBERDI, I. (1995): Informe sobre la situación de la familia en España. Madrid. Ministerio de Asuntos Sociales.

ALBERDI, I. (1993b): "Modelos matrimoniales", en: Del Campo, S. (dir.) Tendencias sociales en España (1960-1990). Vol. 1. Bilbao. Fundación BBV. 
ALBERDI, I. (1997): "La familia. Convergencia y divergencia de los modelos familiares españoles en el entorno europeo", Politica y Sociedad 26, pp. 73-94.

BARBADILLO GRIÑÁN, P. (1995): "Relaciones padres-hijos en familias monoparentales", Infancia y Sociedad 30, pp. 49-54.

CASTRO MARTÍN, T. (1999): "Pautas recientes en la formación de pareja". Revista Internacional de Sociología ( $3^{\text {a }}$ época), pp. 61-94.

CECS (1995): España 1994. Una interpretación de su realidad social. Madrid. Fundación Encuentro.

CIS (1997) Estudio n ${ }^{\circ} 2248$ : Barómetro de Mayo.

CRUZ, P. (1995): Percepción social de la familia en España. Opiniones y Actitudes n 9. Madrid. CIS.

DE MIGUEL, A. (1994): La Sociedad Española, 1993-94. Madrid. Alianza.

DEL CAMPO, S. (1991): La nueva familia española. Madrid. Eudema.

DÍAZ BECERRIL, D. (2004): "Nuevas formas familiares", Poriularia $n^{\prime} 4$, pp. 219-230.

DONALD, M. (1994): "Las familias monoparentales: ¿El modelo familiar del futuro? Cuadernos de Realidades Sociales 43-44, pp. 27-43.

FERNÁNDEZ CORDÓN, J. y TOBÍO SOLER, C. (1998): "Las familias monoparentales en España", REIS 83, pp. 51-85.

FLAQUER, L. (2000): Las politicas familiares en una perspectiva comparada. Barcelona. Fundación "La Caixa".

FLAQUER, L. y SOLER, J. (1990): Permanencia y cambio en la familia española. CIS. Madrid.

GIDDENS, A. (2001): Un mundo desbocado. Los efectos de la globalización en nuestras vidas, Madrid, Taurus.

GONZÁLEZ, M.M. (2000): Monoparentalidad y exclusión social en España. Sevilla. Área de Economía y Empleo. Ayuntamiento de Sevilla.

IGLESIAS DE USSEL, J. (1998): La familia y el cambio politico en España. Madrid. Tecnos.

IGLESIAS DE USSEL, J. (dir.) (1994): "Familia", en: Juárez, M. (Dir.), V Informe sociológico sobre la situación social en España. Sociedad para todos en el año 2000. Madrid. FOESSA.

INE (2002): Movimiento Natural de la Población, www.ine.es (acceso del 7 de octubre de 2002).

MEIL LANDWERLIN, G. (1995): "Presente y futuro de la política familiar en España". REIS 70, pp. 67-90.

MORENO MÍNGUEZ, A. (2000): "Las familias monoparentales en España". Revista Internacional de Sociología (tercera época) 26, pp. 39-63.

MORENTE MEJIAS, F. (1996): "la familia ante la vulnerabilidad de la infancia". Revista Internacional de Sociologia (tercera época) 15, pp. 123-141.

PASTOR RAMOS, G. (1997): Sociologia de la familia. Enfoque institucional y grupal. Salamanca. Ediciones Sígueme.

REQUENA, M. (2001): "Los hogares en Europa, con especial referencia a España", en: INE, Condiciones de vida en España y en Europa. Madrid. INE.

SARRIBLE, G. (1996): "Segunda pareja y diferencias por género". REIS 76, pp. 123-139.

TOBÍO SOLER, C. y FERNÁNDEZ CORDÓN, J. (1999): "Monoparentalidad, trabajo y familia". Revista Internacional de Sociologia (tercera época) 22, pp. 67-97.

VAN DE KAA, D. J. (1987): “Europe's Second Demographic Transition". Population Bulletin $41, n^{0} 1$, pp. $1-57$. 